\title{
ROBOTIC ARM STRUCTURAL FRAME ENHANCEMENT BY GYROID LATTICE CUBE INTEGRATION
}

\author{
Alexandru Stan ${ }^{1}$, Nichita Larisa Milodin ${ }^{1}$ \\ ${ }^{1}$ National Institute of Research and Development for Mechatronics and Measurement Technique - INCDMTM, \\ Bucharest, Romania \\ Emails: alexandrustan71.sa@gmail.com, nichita.milodin@gmail.com
}

\begin{abstract}
The article deals with the mechanical behaviour of a robotic arm having a gyroid lattice cell based structural frame within the arm itself. The gyroid is well know for its poriforous structure that can provide remarkable mechanical properties such as stiffness, strength and substantial energy absorption due to the regular and organic structure. Therefore, the gyroid structures are widespread throughout the various industry palette, such as automotive, aerospace, biomedicine and mechanical industries. Due to its complex structure, the gyroid can not be obtained using the classic technologies, for example milling and turning. The most used technology, especially in mechanical engineering, is the modern additive technology. In this particular case, the gyroid structure is obtained using the selective laser sintering (SLS) additive manufacturing technique. Prior to the manufacturing phase, a designing phase must be conducted starting with a 3D model. Once the final structure has been obtained through 3D designing techniques it is suitable for implementating it within the robotic arm's structural frame. To sum up, in the article have been presented the following: the steps troughout the generative process of the lattice structure, the implementation within the structural frame and manufacturing, including experimental mechanical effort studies.
\end{abstract}

Keywords: Gyroid, Structure, Robotic arm, SLS Additive Manufacturing.

\section{Introduction}

The need for stronger yet light structures has pushed further the research and materials development. In order to achieve this objective, scientist and researchers have contucted studies regarding the architectural cellular materials[1]. The reason why is that when a solid is processed into a foam-like structure, the single-valued properties are vastly extended, such as stiffness, strength, thermal conductivity, diffusivity, electrical resistivity[2].

The gyroid surface belongs to triply periodic minimal surface (TPMS) family and it has been discovered experimentally by Alan H. Schoen in 1960. A TPMS is a minimal surface which is periodic in three independent directions and are described in terms of fundamental patch or asymmetric unit form which the entire surface may be built up to its symmetry elements[4].

A minimal surface is a surface which has a minimum area with zero mean curvature defined by the equation

$$
H=\frac{k_{1}+k_{2}}{2}
$$

at every point, where

$$
k_{1}=\frac{1}{r_{1}}
$$

and

$$
k_{2}=\frac{1}{r_{2}}
$$

represent the principal curvatures, see Fig.1.
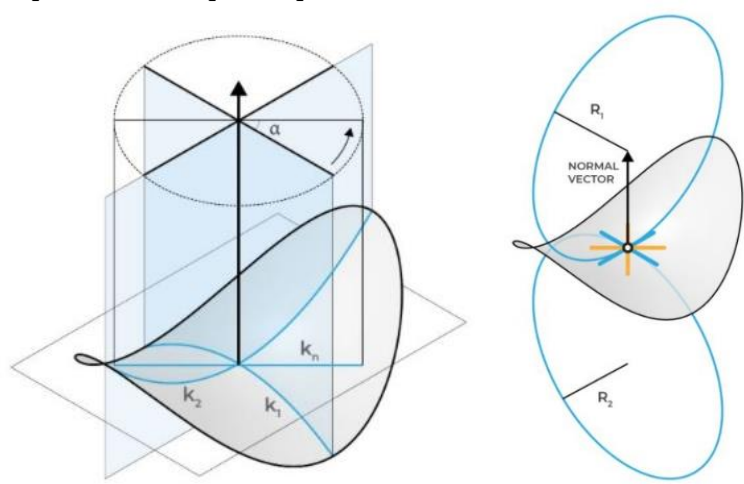

Figure 1 - Directions of the principle curvatures[3]

Therefore, the normal curvature, $k_{n}$, can be calculated for evey direction having a deviation angle $\alpha$.

The normal curvature depending on direction $\alpha$ is described by the following equation:

$$
k_{n}(\alpha)=k_{1} *(\cos \alpha)^{2}+k_{2} *(\sin \alpha)^{2}
$$

where $\alpha$ is always $45^{\circ}$ on minimal surface [3]. 
It is known that eight of these fundamental units are needed in order cu generate a cubic cell of a cubic gyroid structure. After a cubic cell is obtained, the gyroid structure can be built multiplying as many cells as needed in a 3D space. A gyroid structure can be generated by using the following equation:

$\cos x * \sin y+\cos y * \sin z+\cos z * \sin x=0$

Where $x \in\left[-\pi_{v} \pi\right], y \in\left[-\pi_{v} \pi\right]$ and $z \in\left[-\pi_{v} \pi\right]$.

\section{Gyroid Structure Generation 2.1 Fundamental unit generation}

As it was mentioned before, a fundamental unit is needed to be generated in order to build the entire gyroid structure. In order to model the structure, SolidWorks CAD software will be used, Firstly, it has to be sketched within a cube volume, see Fig. 2 .

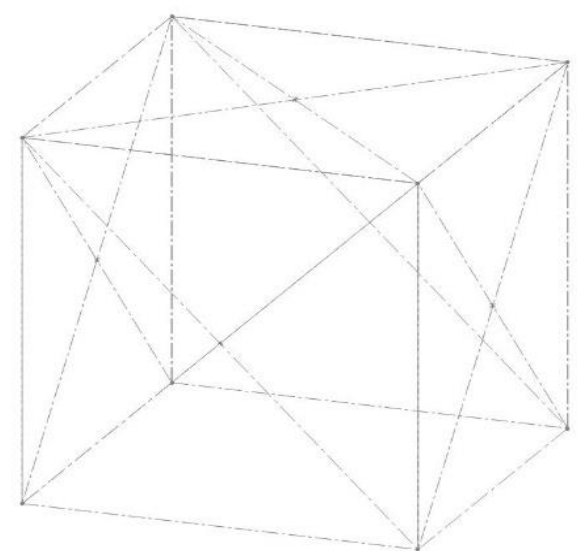

Figure 2 - Cube volume - dimetric view-where side is

$$
l=10 \mathrm{~mm}
$$

The second step lies in running the 6 radiuses, $R=6 \mathrm{~mm}$, of the fundamental unit as follows in Fig. 3 .

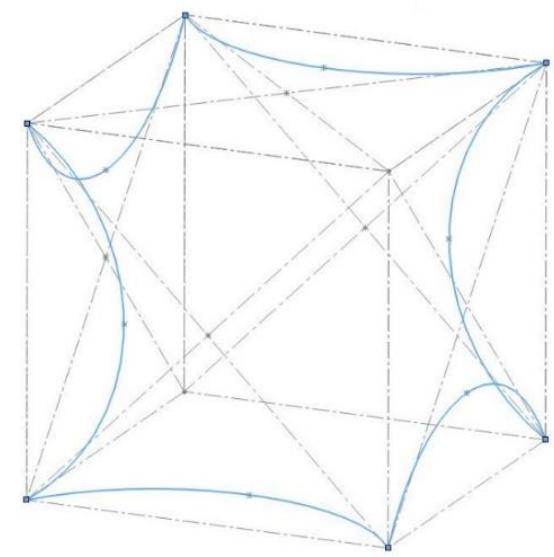

Fig. 3 - The six radiuses of the lattice surface dimetric view

The third step consists in tracing the guidance curvatures. It is mandatory to start tangentially from the maximum of the radiuses on the perpendicular plane and going to a stop in the center of the cube the zero mean curvature - as shown in Fig. 4.

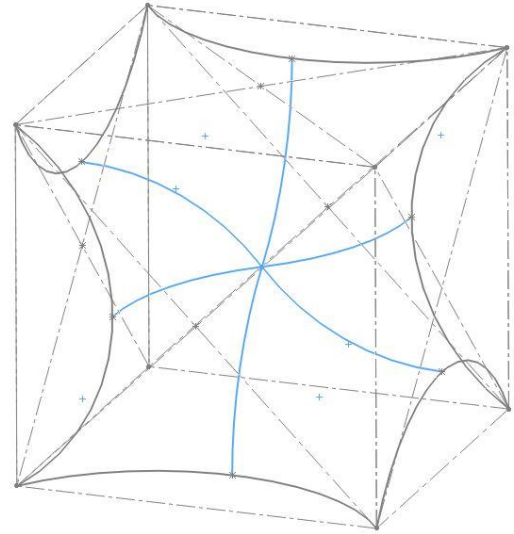

Figure 4-Guidance curvatures - dimetric view

Next, the surface is generated using the Fill feature of SolidWorks, see Fig. 5.

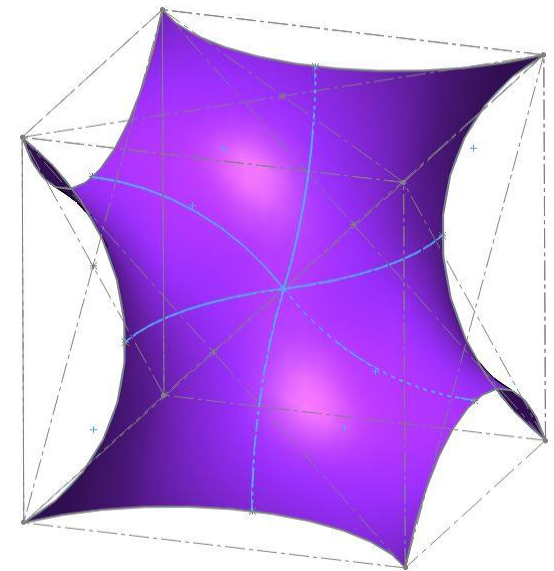

Figure 5 - Fundamental unit surface fill - dimetric view

\subsection{Gyroid cube generation}

Once the fundamental unit is generated, a total of 6 units is needed in order to create a $2 \times 2 \times 2$ gyroid cell by means of rotational symmetry, see Fig. 6.

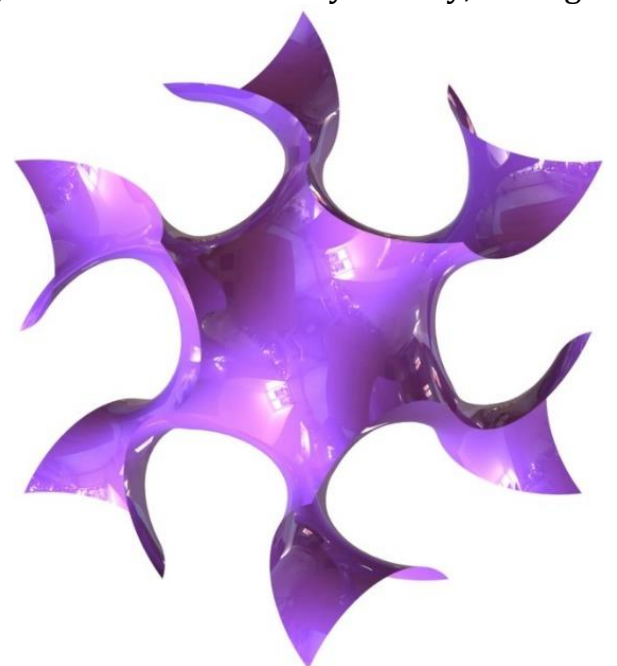

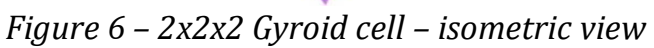


In order to have a workable and strong gyroid structure, the fundamental unit must be transformed into a solid by adding solid volume in order to obtain a solid cell as you can see in Fig. 7 .

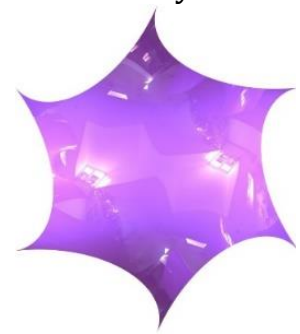

a)

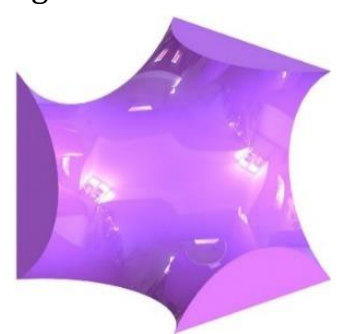

b)
Figure 7 - a) Fundamental unit surface, b) Solid fundamental unit.

Having a solid unit provides a certain thickness that depends on the above radiuses. By doing so we can find out the volume ratio of the solid phase. The volume ratio equation is given by the following equation:

$$
V_{r}=\frac{V_{p h}}{V_{t}} * 100[\%]
$$

Where $V_{\text {ph }}$ is the volume of the solid phase and $V_{t}$ is the total volume of the cube. In this specific case, the total volume of the cube is

$$
V_{\mathrm{t}}=1000 \mathrm{~mm}^{3}
$$

while the

$$
V_{\text {ph }}=499.63 \mathrm{~mm}^{2}
$$

Therefore, using equation (6), the volume ratio is

$$
V_{r}=\frac{499.63 \mathrm{~mm}^{3}}{1000 \mathrm{~mm}^{3}} * 100[\%]=49.963 \%
$$

In conclusion, any structure will be having $\sim 50 \%$ less weight and volume with the given radiuses' values, $R=6 \mathrm{~mm}$, in comparison with the full volume of the same structure.

Having the solid unit, as seen in Fig. 7b), a new solid cell can be created by applying the same rotational symmetry that was applied in the nonsolid cell, see Fig. 8 .

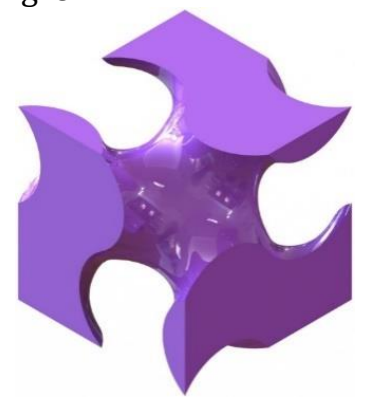

Figure $8-2 \times 2 \times 2$ Solid cell - isometric view

Once the solid cell is complete, we can proceed to the generation of the solid gyroid cube by linearly patterning solid cells along the $\mathrm{x}, \mathrm{y}$ and $\mathrm{z}$ axis. As a result, a $3 \times 3 \times 3$ gyroid cube is obtained as shown in Fig. 9.

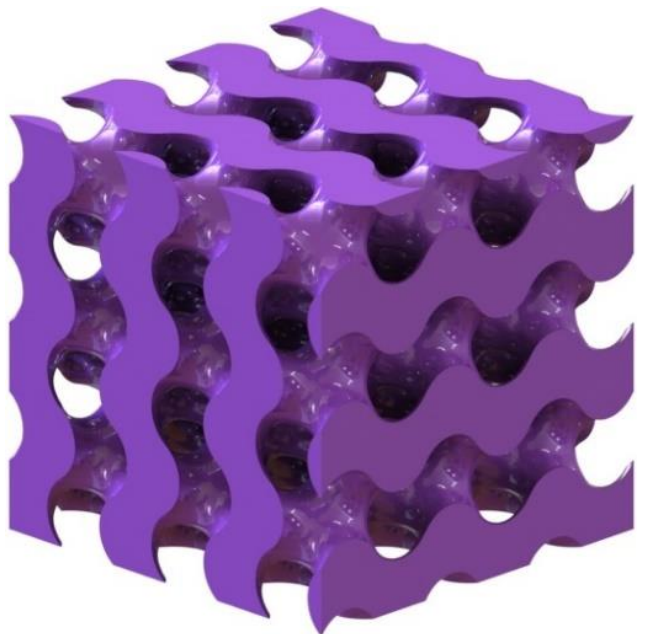

Figure $9-3 \times 3 \times 3$ Gyroid cube - trimetric view

As soon as the gyroid cube is complete, we can proceed with building the adequate volume of the reinforcement structure as needed.

\section{Gyroid Structure Deployment}

Firstly, in the following figure is presented the entire 2-link arm assembly, highlighted with blue, that will be modified see Fig. 10.

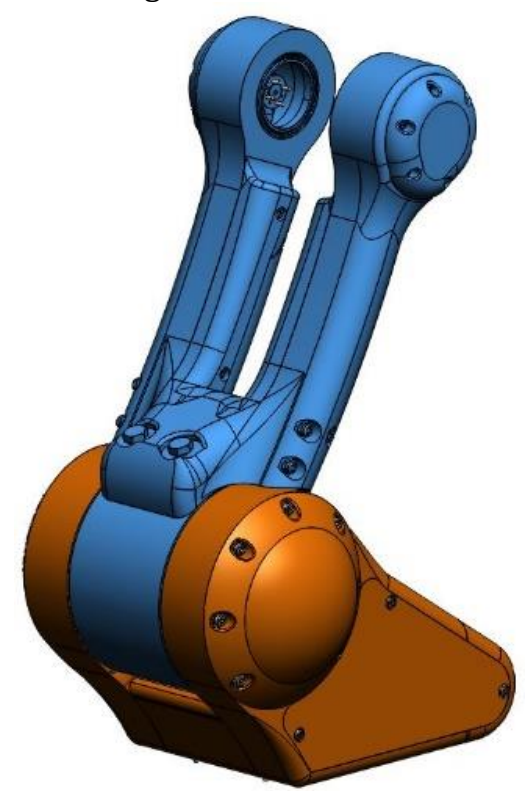

Figure 10 - 2-link arm assembly - dimetric view

Secondly, the section that will be cut out from the robot's arm link must be marked-out, see Fig. 11a) and Fig. 11.b).

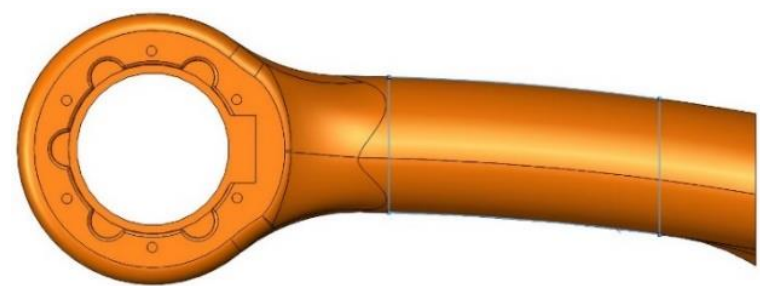

Figure 11.a) Cut-out gray perimeter - side-view 

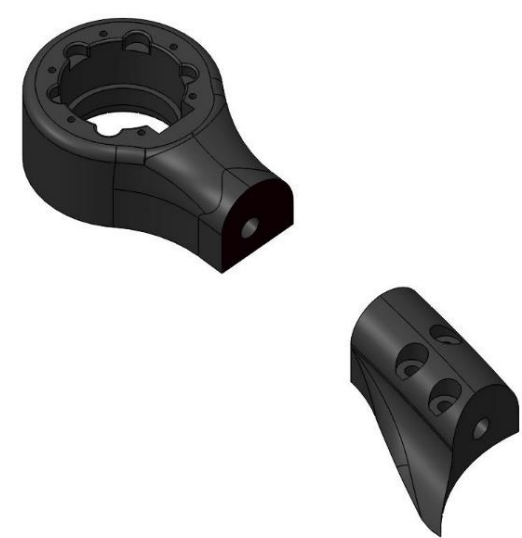

Figure 11.b) Link after being cut - isometric view

Next, the new gyroid structure must be sculpted after the link's shape in order to fit perfectly in the empty section. Beforehand, a gyroid volume bigger than the cut-out volume but be created in order the have disposable volume, see Fig. 12.

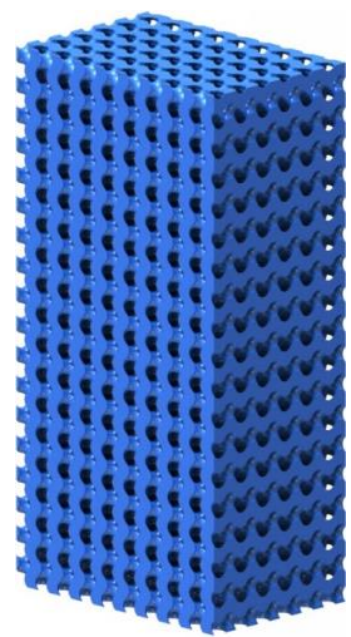

Figure 12 - Rectangular gyroid prism

Once the new structure is obtained Fig. 13, it is ready to be integrated within the link's body as shown in Fig. 14.

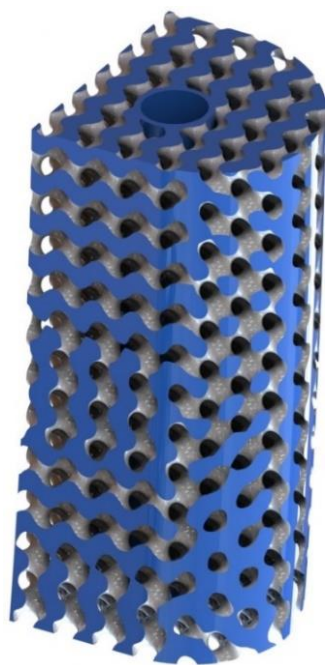

Figure 13 - New structural frame

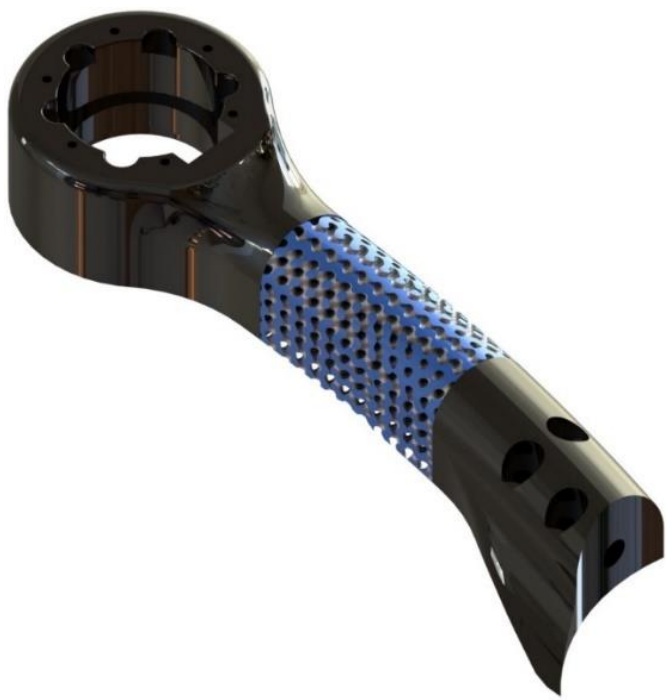

Figure 14 - Final link assembly - rendered

\section{Manufacturing and Testing 4.1 Manufacturing phase}

Prior flexural testing, the enhanced link must be manufactured. Being a 3D designed prototype and taking into account the fact that we are dealing with a very complex structure, the most reliable and fastest manufacturing method that can be chosen is the SLS additive manufacturing.

In this particular case, the printer FORMIGA P110 will be used to manufacture the arm's link using PA 2200 Nylon material.

After the experimental link is complete, it was sprayed black, except the gyroid section, which was sprayed blue in order to stand out from the rest of the part, see Fig. 15 and Fig. 16.

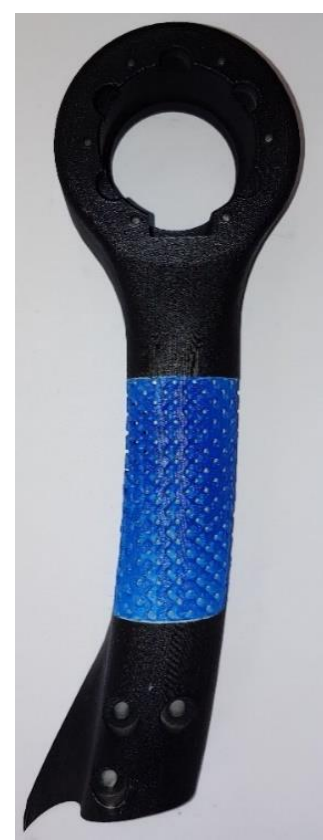

a)

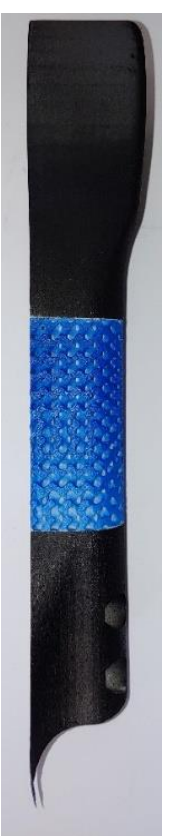

b)
Figure 15 - a) Link - side-view, b) Link - front-view 


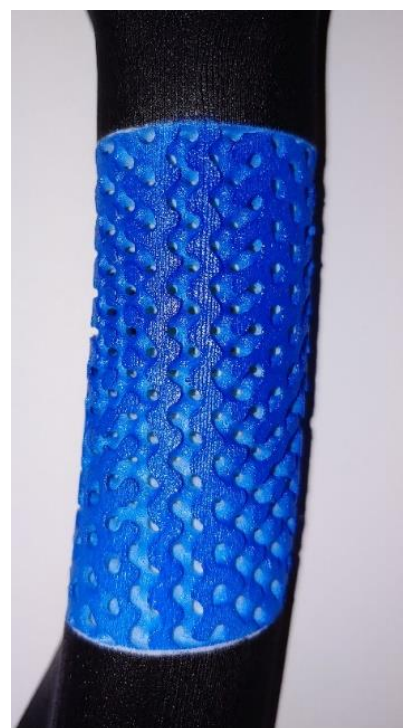

Figure 16 - Gyroid structure close-up

\subsection{Testing phase}

The main stress-wise aspect relevant to this case is the flexural stress behaviour of the part while being in the most adverse positon for a robotic arm, parallel to the horizontal plane. Unfortunately, due to the prototype's very complex geometry, SolidWorks was not able to create a mesh in order to simulate the part. Therefore, the experimental testing method is approached as follows.

Furthermore, the experimental stand is shown in Fig 17. In order to fix in place the experimental part, a jaw vice had to be mounted on the base plate of the flexural testing equipment.

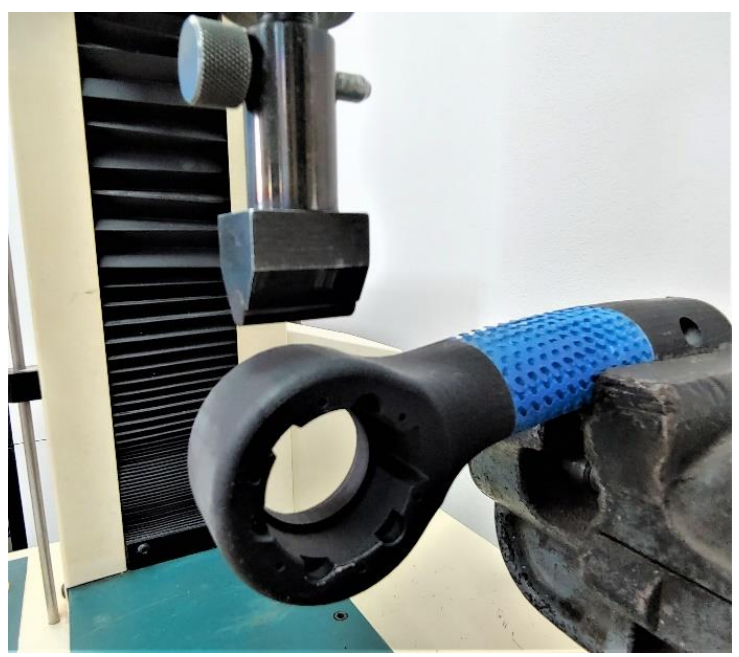

Figure 17 - Testing stand

Next, the study will be conducted using a stress force normal to the part, $F_{\max }=800 \mathrm{~N}$, the maximum force that machine can provided by the machine. The main objective of the study is to observe the mechanical behaviour at a force threshold, $F_{t h}=100 \mathrm{~N}$, the maximum force the assembly has to withstand in a real circumstance and the maximum force the assembly can withstand before yielding. As a preliminary note, it is know that the flexural strength of PA2200 Nylon is $\sigma_{f s}=58 \mathrm{Mpo}$ [5].

In the following figure, it is shown the part's position while being subjected to a gradual increasing normal force up to $F_{\max }=800 \mathrm{~N}$.

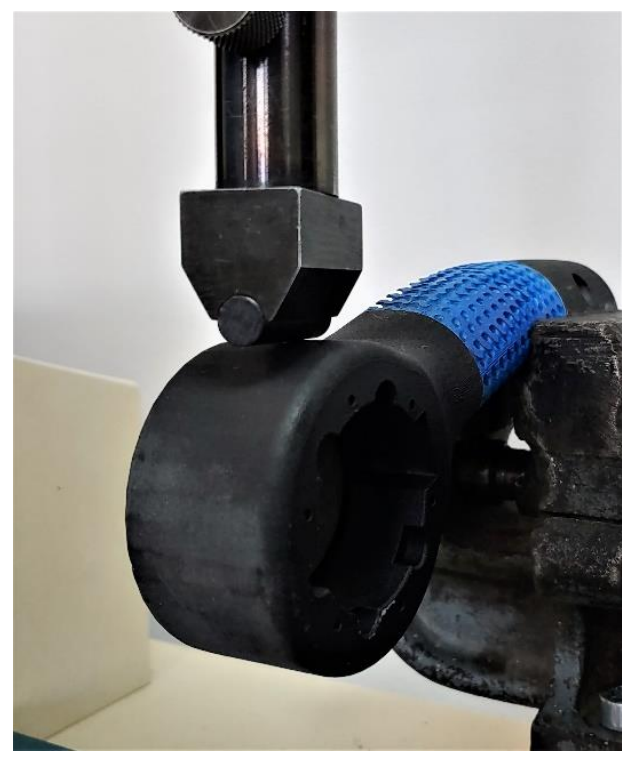

a)

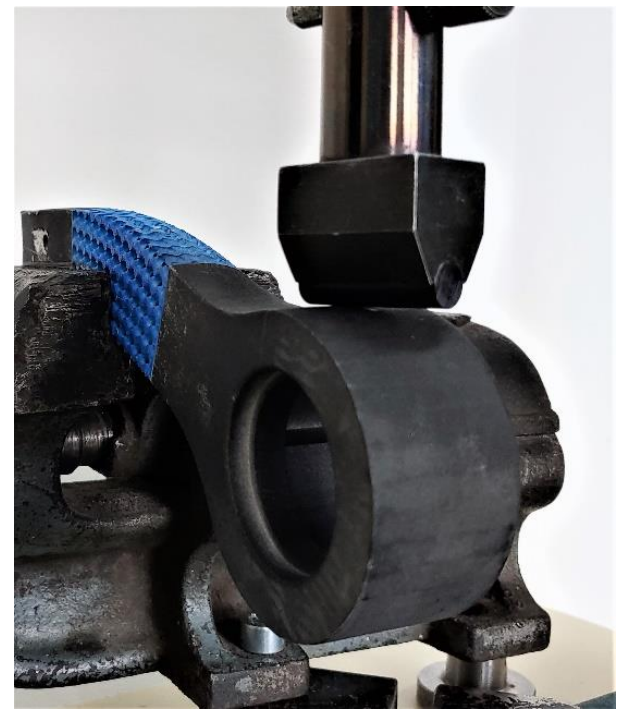

b)

Figure 18 - a) Maximum flexural stress - $1^{\text {st }}$ view, b)Maximum flexural stress $-2^{\text {nd }}$ view

As shown in the plot below, Fig. 19, the black plot line describes the dependency of the displacement towards the applied force and the red dotted line represents the linear relationship, ideal behaviour, between the normal force and displacement.

As it was previously stated, the force threshold of interest is $F_{t h}=100 \mathrm{~N}$ and also the dependent displacement d. As the graph illustrates, at $F_{\text {th }}=100 \mathrm{~N}$, the system outputs a displacement $d_{1}=4 \mathrm{~mm}$. 
The highlight of this experiment was the unforeseen performance of the prototype registered at the $F_{\max }=800 \mathrm{~N}$, where the system outputs a displacement $d_{1 \max }=27 \mathrm{~mm}$. In addition to this, it must be pointed out the fact that the prototyped part remained in the proportional domain, given the linear nature of the plot line growth, until $F_{\text {prop }}=550 \mathrm{~N}$ was reached and then gradually closing in on the yield point, but never reaching it. Therefore, the prototype stayed within the elastic deformation domain once the force was removed. The mechanical behaviour can be seen in the diagram below, see Fig. 19.

In order to be able to compare these results towards the non-gyroid part, a simulation experiment must be conducted with this part.
By replicating as best as possible the practical testing conditions in the CAD software, the results are shown in Fig. 20 and Fig. 21.

As seen in Fig 20b) and Fig. 21b), the system outputs a displacement $d_{2}=1.51 \mathrm{~mm}$ at $F_{\text {th }}=100 \mathrm{~N}, d_{2_{\text {max }}}=11.87 \mathrm{~mm}$ at $F_{\max }=800 \mathrm{~N}$ and maximum stress of $\sigma_{\max }=51_{s} 07 \mathrm{Mpa}$. Knowing that $\sigma_{f s}=58 \mathrm{Mpa}$, the original part can withstand greater forces than predicted.

Comparing the following two sets of displacements, $d_{1}=4 \mathrm{~mm}$ to $d_{2}=1.51 \mathrm{~mm}$ and $d_{1_{\max }}=27 \mathrm{~mm}$ to $d_{2_{\max }}=11.87 \mathrm{~mm}$ it can be stated that the protoyped part is more flexible than the original part due to the curvatures within and cell-like gyroid structure.

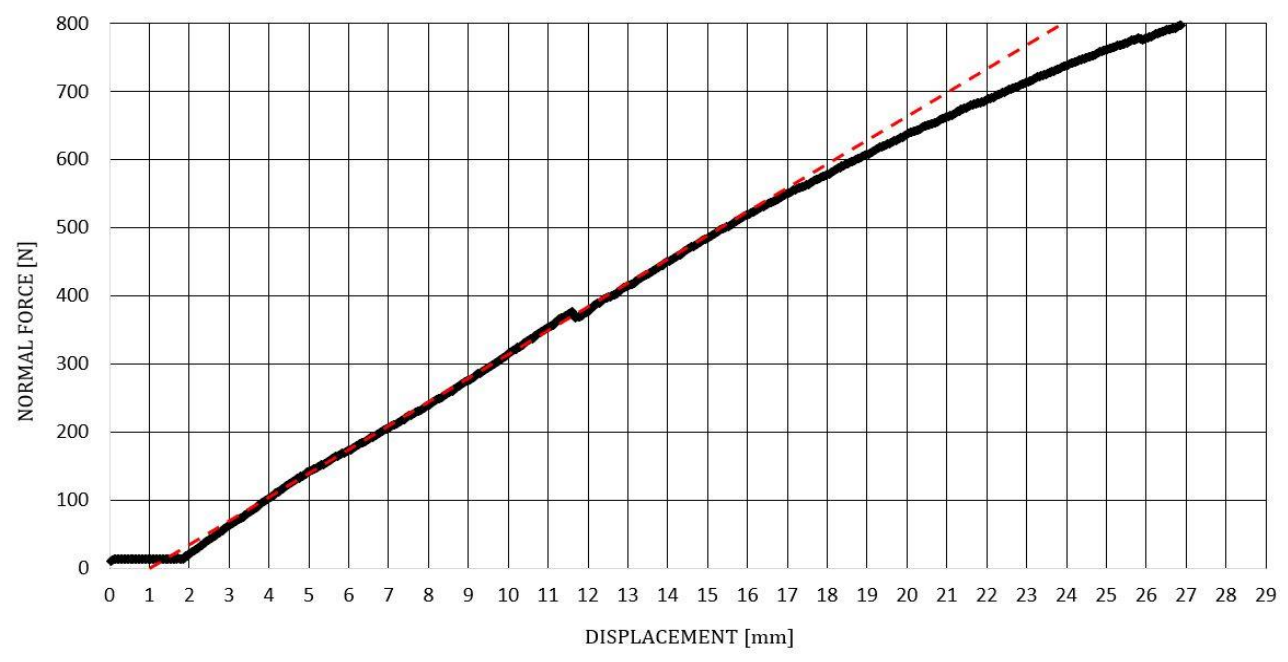

Figure 19 - Normal force - displacement plot

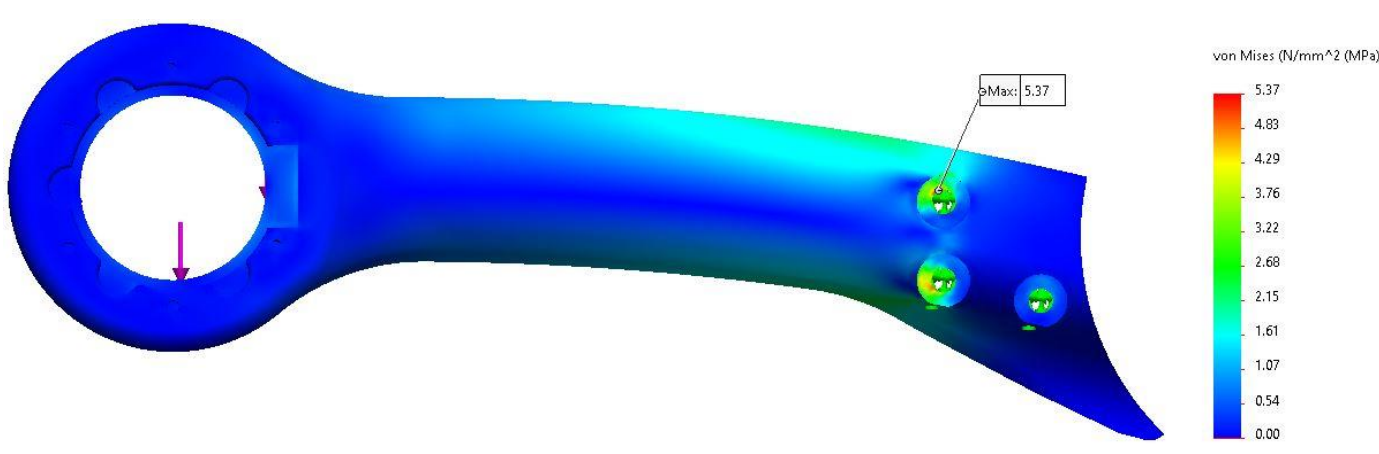

Figure 20.a) $-F_{\text {th }}=100 \mathrm{~N}$ - flexural stress

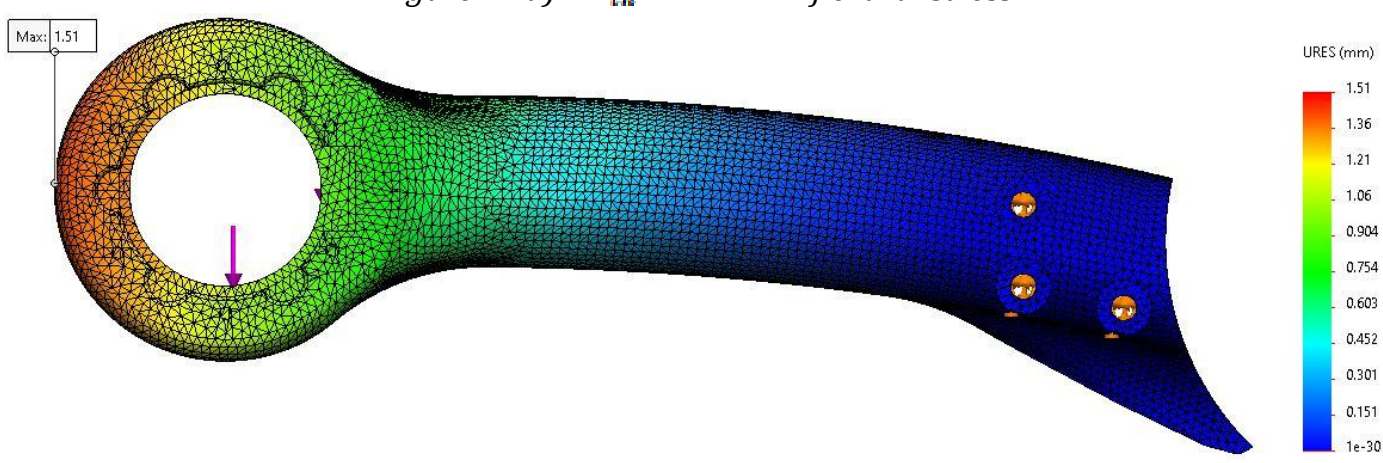

Figure 20.b) $-\boldsymbol{F}_{\text {th }}=100 \mathrm{~N}-\boldsymbol{d}_{2}$ displacement 


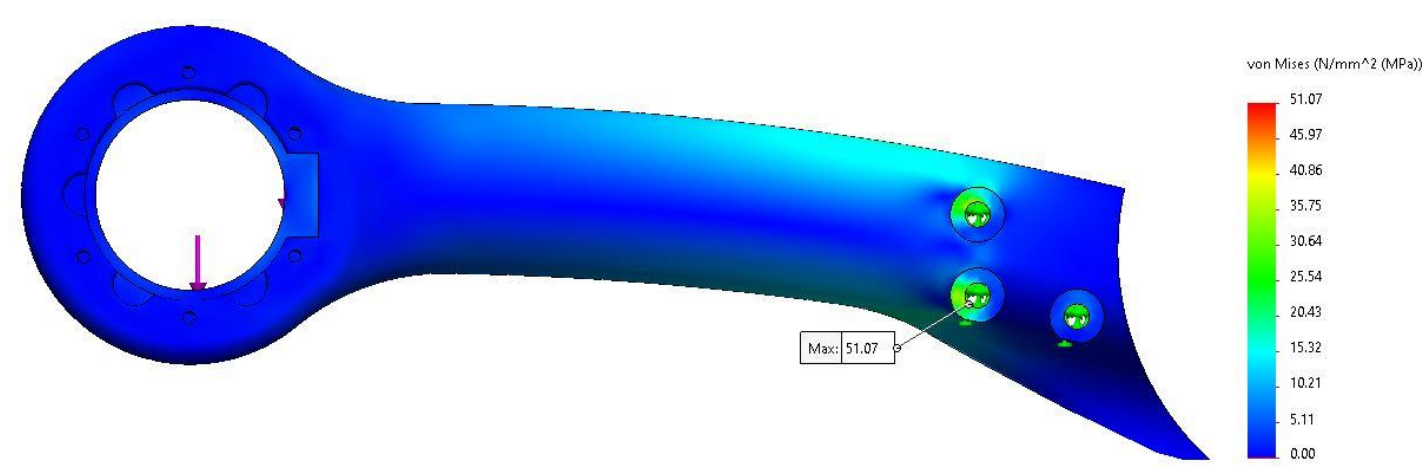

Figure 21.a) $-\boldsymbol{F}_{\max }=\mathbf{8 0 0} \mathbf{N}$ - flexural stress

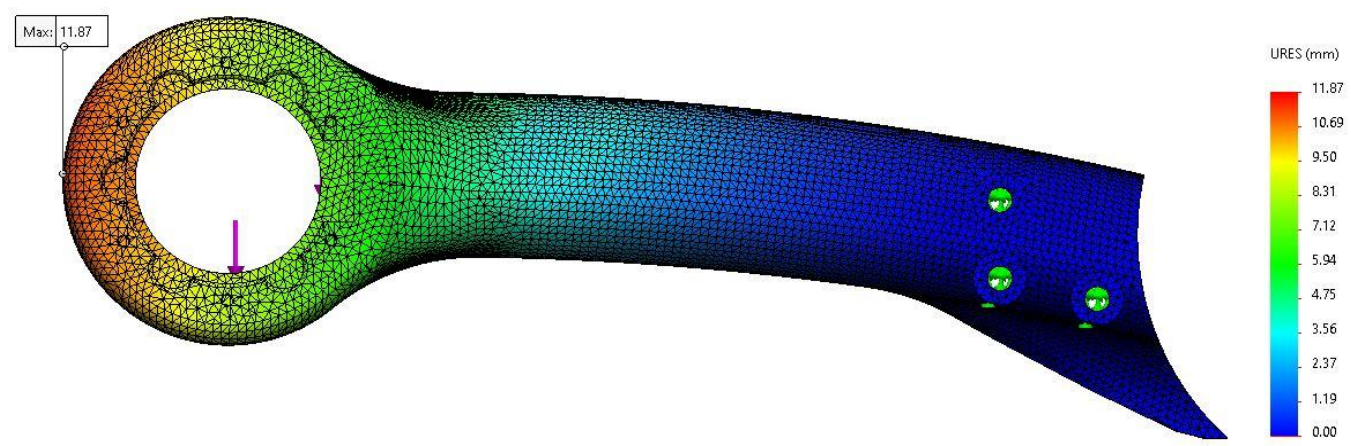

Figure $21 b)-F_{\max }=800 \mathrm{~N}-\boldsymbol{d}_{2_{\max }}$ displacement

\section{Conclusions}

This paper has as primary goal the study of mechanical behaviour of a hybrid robotic arm while being subjected to flexural stress. The hybrid nature is given by the integration of a gyroid-based structure within the arm's structure.

A gyroid-based structure is not suitabile for applications that require repetitive precision due to its increased elasticity compared to a solid that has the same dimensions. The main disadvantage is the stiffness loss of the system.

The main advantage is related to weight and inertial moment reduction while maintaining the strength of the system.

Therefore, the gyroid structure is well suited when implemented in robotic systems that are remotely controlled, need to handle relatively small loads, $\sim 4-5 \mathrm{~kg}$ and are conducting non-repetitive processes.

\section{Acknowledgements}

This work was supported by a grant of the Romanian Ministry of Research and Innovation, Research program NUCLEU, contract no. 17N/2019, project number PN 19.24.01.01.

\section{References}

[1] M.F. Ashby, H. Shercliff, D. Cebon: Materials: Engineering, Science, Processing and Design, Butterworth-Heinemann, 2013.

[2] M.F. Ashby: The properties of foams and lattices, Cambridge, UK, 2005.

[3] https://wewanttolearn.wordpress.com/tag/gyro id/?fbclid=IwAR2R6X9rosUUHX0-

Ene1TtD3UqH6lIZQc5DVCNIBqE7Pwrn1BzZQ1k $5 \mathrm{vOF} 8$

[4] Schoen, A. H.: Reflections concerning triplyperiodic minimal surfaces, Interface Focus, 2(5), (2012).

[5] http://www.shapeways.com/topics/udesign/ma terials/white_strong_flexible/pa2200_material_d ata_sheet_12_08_en_.pdf 